\title{
A Portable Smartphone-based Platform with an Offline Image- processing Tool for the Rapid Paper-based Colorimetric Detection of Glucose in Artificial Saliva
}

\author{
Tansu GölCEZ,* Volkan KILIÇ, ${ }^{* * \dagger}$ and MuSTAfa ŞEN***† \\ *Biomedical Technologies Graduate Program, Izmir Katip Celebi University, Izmir, 35620, Turkey \\ **Department of Electrical and Electronics Engineering, Izmir Katip Celebi University, Izmir, 35620, Turkey \\ ***Department of Biomedical Engineering, Izmir Katip Celebi University, Izmir, 35620, Turkey
}

\begin{abstract}
In this study, a microfluidic paper-based analytical device ( $\mu$ PAD) was integrated with a smartphone app capable of offline (without internet access) image processing and analysis for the rapid colorimetric detection of glucose. A selfinking stamp was used to form hydrophobic channels on a piece of paper-towel due to its superior water absorption efficiency. As demonstrated, the developed sensor was employed for the colorimetric detection of glucose in artificial saliva in the linear scope of $0-1 \mathrm{mM}$ with a calculated detection limit of $29.65 \mu \mathrm{M}$. The experimental results show that the quantitative analysis of glucose with the proposed smartphone platform could be completed in less than one minute. The app developed for the smartphone platform is capable of extracting the color-changing area with an embedded image processing tool which could address the problem of color uniformity in the detection zones of $\mu$ PAD. The integrated platform has great potential to be used for non-invasive measurements of glucose in body fluids, like tears, sweat and saliva.
\end{abstract}

Keywords Glucose detection, smartphone colorimeter, image processing, Android application, $\mu$ PAD

(Received July 16, 2020; Accepted September 24, 2020; Advance Publication Released Online by J-STAGE October 2, 2020)

\section{Introduction}

Paper-based sensors as novel analytical tools have great potential to be used in a variety of fields ranging from environmental monitoring to clinical and point-of-care tests. These sensors have many advantages such as being disposable, practical, costeffective, and user-friendly. ${ }^{1}$ The first step in the prevention and treatment of a disease is diagnosis. According to the World Health Organization (WHO), in order for diagnostic devices to be used in developing countries, they are expected to meet the following criteria, which are abbreviated as "ASSURED": being affordable, sensitive, specific, user-friendly, rapid and robust, equipment-free and deliverable to end-users. ${ }^{2,3}$ Paper-based sensors are thought to have the potential to meet these specified criteria. The concept of fabricating microfluidic paper-based analytical devices ( $\mu$ PAD) has attracted an increasing amount of attention after being introduced by Martinez et al., ${ }^{4}$ and has become popular in the detection of multiple analytes due to its potential applications in $e . g$. analytical and clinical chemistry. ${ }^{5,6}$

Various detection methods such as chemiluminescence, fluorescence, electrochemical and colorimetric detection have been applied to $\mu$ PADs for rapid, sensitive and selective analysis. ${ }^{7,8} \quad$ Among these detection methods, colorimetric detection has emerged as a useful tool for rapid qualitative

$\dagger$ To whom correspondence should be addressed. E-mail: volkan.kilic@ikcu.edu.tr(V. K.); mustafa.sen@ikcu.edu. $\operatorname{tr}($ M. Ş.) analysis due to its ability to be used in remote locations or those with poor infrastructure where sophisticated tools or timeconsuming steps cannot be afforded. ${ }^{9}$ Moreover, the presence of the analyte in the detection zone causes a color change, enabling visual readout, which does not require special equipment for analysis and makes the system cheaper. The use of colorimetric detection in paper-based analysis is promising as it enables portable, rapid and cost-efficient analysis with low sample and reagent consumption. However, this technology has some limitations in practice, like poor color uniformity. This problem might stem from the case that the solution flowing through the microchannels carries both the colorimetric reagents and unbound enzymes at the detection zone, which degrades the color homogeneity. Various techniques have been introduced to improve the color uniformity to a certain extent, such as the incorporation of chitosan on cellulose,$^{10}$ paper oxidation ${ }^{11}$ and fluid flow control. ${ }^{12}$

Detection in most of the commercially available colorimetric diagnostic tools, including pregnancy and urine test strips, depends on visual inspection of any discoloration in the detection zone. Visual inspection does not provide quantitative data due to poor accuracy. The easiest way for the quantification of a substance is to capture the visible color change using a camera, and to measure the color intensity using imaging software. Due to the latest advances in camera and sensor technologies, existing smartphones are nowadays fabricated with a low-power, high-performance processor and a highresolution digital camera for image capturing, even in dark conditions, which has led them to be used in quantitative 


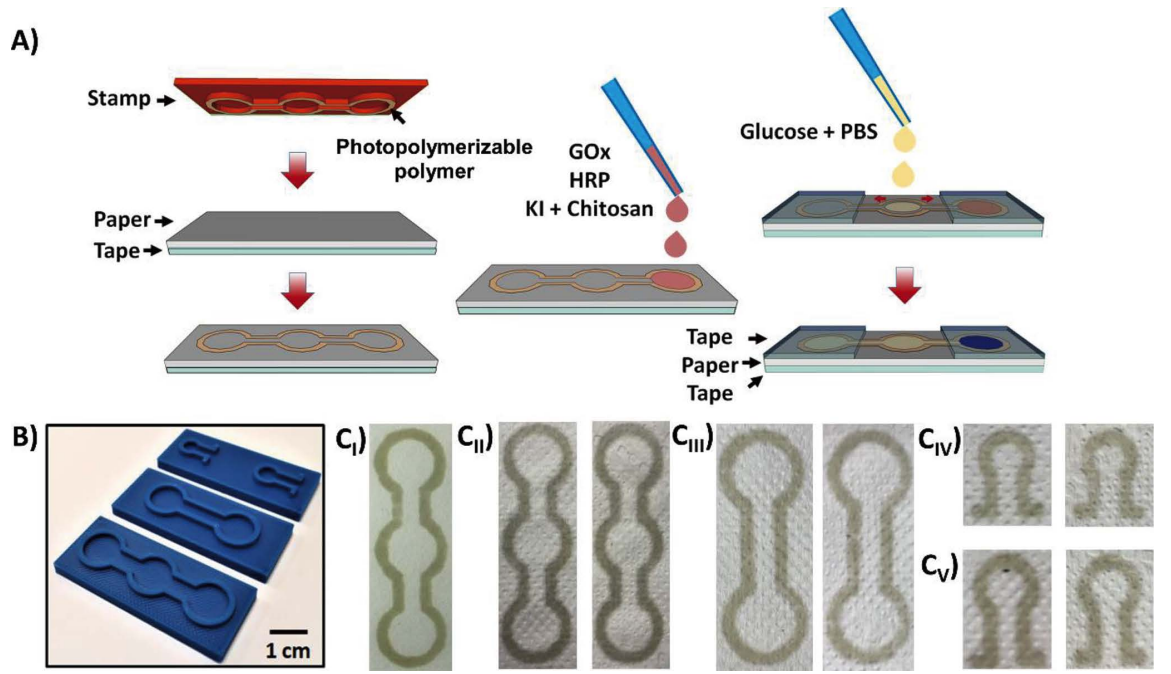

Fig. 1 Schematic illustration of the fabrication process used to form hydrophobic channels on a piece of paper (A). 3D-printed molds (B) used with a self-inking stamp to form hydrophobic channels in different shapes (C). A light-curable polymer hydrophobic in nature was used as ink and diffused to both sides of the paper $\left(\mathrm{C}_{\mathrm{II}}, \mathrm{C}_{\mathrm{III}}, \mathrm{C}_{\mathrm{IV}}, \mathrm{C}_{\mathrm{V}}\right)$.

analysis. ${ }^{13,14}$ Smartphone colorimeters are used in sample quantification based on color change with respect to the concentration, as in peroxide quantification ${ }^{15}$ or with respect to time as in methylene blue degradation. ${ }^{16}$ Recently, researchers have proposed several smartphone colorimeter designs, driven by applications such as water quality sensing, ${ }^{17} \mathrm{pH}$ detection,,${ }^{18}$ glucose sensing, ${ }^{19}$ bisphenol-A detection ${ }^{20}$ and food allergen testing. ${ }^{21}$ In these designs, the smartphone colorimeters need to extract the color information depending on the color variation using color components of alternative color spaces like RGB (Red-Green-Blue), HSV (Hue-Saturation-Value), and L*a*b* (Lightness, Green-Red, Blue-Yellow) for quantitative color analysis. ${ }^{22-24}$ In addition to color spaces, Joint Photographic Experts Group (JPEG) and RAW image formats were also studied to analyze the impact of the formats in the colorimetric analysis. ${ }^{16,25}$ In this study, a $\mu$ PAD was used with a portable smartphone platform for the colorimetric analysis of glucose.

A photopolymerizable polymer as a printing material was used to print the $\mu$ PAD on a paper towel with the help of a 3D-printed mold (Fig. 1A). Subsequently, HRP/GOx enzymes, chitosan and KI were placed in the detection zone for the colorimetric detection of glucose. Glucose at various concentrations was tested and the visible color change was captured using the portable smartphone platform. According to the results, the sensor response was linear in the range of 0 to 1 $\mathrm{mM}$ glucose, and analysis could be completed in less than 1 $\min$.

The major contributions of this paper can be summarized as follows:

1. A simple, cost-efficient, and sensitive smartphone-based platform integrated $\mu \mathrm{PAD}$ for the rapid detection of glucose was reported.

2. A self-inking stamp along with a light-curable polymer hydrophobic in nature was used to form hydrophobic channels on a paper-towel.

3. The lateral flow speeds on the paper-towel were compared to that of filter paper.

4. An Android app, named as GlucoSense, capable of offline (without internet access) image processing for precise extraction of the region of interest (ROI) was developed to make the platform more user-friendly for quantitative glucose analysis. Precise extraction of the ROI can address poor color uniformity, and thus improve the accuracy of the analysis.

5. The smartphone-based platform integrated $\mu \mathrm{PAD}$ demonstrated high sensitivity $(\mathrm{LOD}=29.65 \mu \mathrm{M})$ and selectivity for glucose detection in artificial saliva. Therefore, it has great potential for non-invasive measurements of glucose in body fluids like tears, sweat and saliva.

\section{Materials and Methods}

\section{Materials}

Phosphate buffered saline (PBS) (Sigma Aldrich, USA), glucose oxidase from Aspergillus niger $(211 \mathrm{U} / \mathrm{mg}$ ) (Sigma Aldrich, USA), peroxidase from horseradish (HRP, $325 \mathrm{U} / \mathrm{mg}$ ) (Sigma Aldrich, USA), potassium chloride (KCl) (Sigma Aldrich, USA), potassium iodide (KI) (Sigma Aldrich, USA), chitosan-low molecular weight (Sigma Aldrich, USA), acetic acid-2\% (v/v) in $\mathrm{H}_{2} \mathrm{O}$ (Sigma Aldrich, USA), D(+)-glucose $\left(\mathrm{C}_{6} \mathrm{H}_{12} \mathrm{O}_{6}\right)-\geq 99.5 \%$ (GC) (Sigma Aldrich, USA), paper-towel (Eagle photocell towel, Eagle Professional, Turkey), Self-inking stamp (Kaşem, Turkey), methacrylic/acrylic resin (HTM140 V2, EnvisionTEC, USA), thermoplastic filament (Z-ABS, Zortrax, USA), Whatman qualitative filter paper-grade 1 (Sigma Aldrich, USA), artificial saliva (NeutraSal, Germany).

\section{Formation of hydrophobic channels on paper}

Various stamps were designed using 3D CAD design software (SolidWorks version 2019, Dassault Systemes, USA) and printed with high precision using a 3D printer (M200, Zortrax, USA) with a $1.7 \mathrm{~mm}$ thick thermoplastic filament (Fig. 1B). The four designs have different dimensions. The design in Figs. $1 C_{I}$ and $1 C_{I I}$ has a $45.58 \mathrm{~mm}$ length and each circle has a $5 \mathrm{~mm}$ inner radius. The center to center distance for the circles is $17 \mathrm{~mm}$. The design in Fig. $1 \mathrm{C}_{\text {III }}$ has $37 \mathrm{~mm}$ in length. The circle dimensions are the same as Figs. $1 \mathrm{C}_{\mathrm{I}}$ and $1 \mathrm{C}_{\mathrm{II}}$, however, the center-to-center distance for the circles is $24 \mathrm{~mm}$. The 
channel width and thickness of both designs (Figs. 1C $\mathrm{C}, 1 \mathrm{C}_{\mathrm{II}}$, and $\left.1 \mathrm{C}_{\mathrm{III}}\right)$ are 3.70 and $1.5 \mathrm{~mm}$, respectively. The design length, channel length and the radius of the inner circle in Fig. $1 \mathrm{C}_{\mathrm{IV}}$ are $10.4,5.35$ and $3.75 \mathrm{~mm}$, respectively. The design length, channel length and the radius of the inner circle in Fig. $1 \mathrm{C}_{\mathrm{V}}$ are $12.3,6.4$ and $4.7 \mathrm{~mm}$, respectively. The channel width and thickness of both designs (Figs. $1 \mathrm{C}_{\mathrm{IV}}$ and $1 \mathrm{C}_{\mathrm{V}}$ ) are 3 and $1.5 \mathrm{~mm}$, respectively.

The 3D-printed stamp was then mounted onto the base of a self-inking stamp (maximum impression area: $60 \times 25 \mathrm{~mm}$ ) using double-sided tape. The ink cartridge of the self-inking stamp was filled with a hydrophobic light-curing methacrylic/ acrylic resin and inserted into the self-inking stamp. The designed stamp was used to cast the resin on a paper-towel and filter paper (Whatman No. 1) to form hydrophobic channels (Fig. 1A). Subsequently, the resin on both papers was lightcured for $20 \mathrm{~s}$ in a post-curing apparatus that delivered light in the $390-420 \mathrm{~nm}$ wavelength range (PCA 100, EnvisionTEC, USA). Next, the lateral flow behavior in the hydrophobic channels formed on paper-towel and filter paper was assessed using a PBS solution colored with black ink at ambient temperature $\left(25^{\circ} \mathrm{C}, 45 \% \mathrm{RH}\right)$. First, the printed device was cut proportionally using a pair of scissors and completely sealed with transparent tape, except for the sample insertion. Then, $30 \mu \mathrm{L}$ aliquots of colored solution were added to the sample insertion region of the device and the lateral flow was recorded using a smartphone camera.

\section{Fabrication of $\mu P A D$ and glucose detection}

The detection zones of $\mu$ PADs (Fig. 1C $\mathrm{C}_{\text {II }}$ ) were modified for the colorimetric detection of glucose. A detection mixture containing $180 \mathrm{U} / \mathrm{mL}$ GOx, $50 \mathrm{U} / \mathrm{mL}$ HRP, $3 \mathrm{mM} \mathrm{KI}$ and $1 \%$ $(\mathrm{w} / \mathrm{v})$ chitosan was prepared in PBS at $\mathrm{pH}$. Then, $5 \mu \mathrm{L}$ aliquots of the mixture were carefully and slowly injected into the detection zone of $\mu$ PADs (Fig. 1A). The mixture was dried in the air for about $10 \mathrm{~min}$ at room temperature. Next, both sides of $\mu \mathrm{PAD}$ except, for the region for sample insertion, were sealed with transparent tape. The colorimetric behavior of $\mu \mathrm{PAD}$ was assessed using PBS + glucose solutions. First, the contribution of GOx, HRP and chitosan to the discoloration in the detection zones was demonstrated by removing them one by one from the detection mixture. Basically, three mixtures each lacking one of the aforementioned components were prepared and added to the control regions of $\mu$ PADs. The color change in the control regions of $\mu$ PADs was assessed using $30 \mu \mathrm{L}$ aliquots of PBS solutions containing $10 \mathrm{mM}$ glucose. The color change in the detection and control zones were imaged $1 \mathrm{~min}$ after the test solution was introduced into these regions. Then, $30 \mu \mathrm{L}$ aliquots of test solutions containing glucose at varying concentrations $(0.1,0.25,0.5,0.75,1,5,10,15$, and $25 \mathrm{mM})$ were introduced into the sample insertion regions of $\mu$ PADs and allowed to reach both control and detection zones under lateral flow. Lastly, a sensor with a smaller size and different design (Fig. $1 \mathrm{C}_{\mathrm{IV}}$ ) was used to detect glucose at varying concentrations $(0.1,0.25,0.5,0.75$, and $1 \mathrm{mM})$ in NeutraSal's artificial saliva. Due to its small size, $2 \mu \mathrm{L}$ aliquots of the detection mixture were carefully and slowly injected into the detection zone of $\mu$ PADs and allowed to dry at room temperature ( $10 \mathrm{~min})$.

\section{Selectivity tests}

The selectivity of the paper-based sensor was evaluated in the presence of a number of interfering species such as sucrose $(0.5 \mathrm{mM})$, urea $(0.5 \mathrm{mM})$, lactate $(0.5 \mathrm{mM}), \mathrm{NaCl}(10 \mathrm{mM})$, $\mathrm{KCl}(10 \mathrm{mM})$, and $\mathrm{CaCl}_{2}$. The selectivity tests were conducted in artificial saliva using the device shown in Fig. $1 \mathrm{C}_{\mathrm{IV}}$.

\section{Image processing}

A $3 \mathrm{D}$ printed apparatus was used with a smartphone to take photos of $\mu$ PAD for image processing and quantitative analysis. As stated above, first $30 \mu \mathrm{L}$ aliquots of glucose + PBS solutions at varying concentrations $(0.1,0.25,0.5,0.75,1,5,10,15$, and $25 \mathrm{mM}$ ) were introduced into the sample insertion regions of $\mu$ PADs and then images of these $\mu$ PADs were taken at 10,20 , 30, 40, 50, and 60 s time points using LG G4 (LG, South Korea) smartphone rear-facing camera with the specifications of $1 / 2.6$ inch sensor size, $5312 \times 2988$ pixel resolution and $1.12 \mu \mathrm{m}$ pixel size. To maintain consistency and repeatability, the smartphone camera was used in the manual mode and settings for the ISO level (2200), shutter speed (1/10 s), focus level (f1.8) and white balance were kept constant during imaging. The LG G4 was purposely chosen because it is one of the few smartphones that support both JPEG and RAW file formats. A RAW image contains linear RGB information about the scene radiance, as it is minimally processed data from the digital camera sensor. The RAW images are converted to a commonly used format, like JPEG, for storage after applying postprocessing methods, such as white balance, gamma correction, color space correction, and compression. The JPEG format is usually preferred because it primarily requires low storage space and contains high-image quality. It has also some drawbacks, like missing information due to compression, having non-linear RGB values and providing only 8-bit images.

In this study, the images were processed both in RAW and JPEG formats to analyze the impact of formats on quantitative evaluations. RAW images were converted to the tagged image file format (TIFF) using freely available DCRAW software, ${ }^{26}$ which allows one to reach $\mathrm{R}, \mathrm{G}, \mathrm{B}$ values while preserving the linear relationship between the RAW images and the radiance scene. The RGB images in both the RAW and JPEG formats are converted to HSV and L*a*b* color channels, which are more robust to any illumination variation.

An image dataset, created by capturing nine concentration values at six-time points, was transferred to a computer to process in MATLAB (MathWorks, MA, USA) environment. The region of interest (ROI) in the detection zone, which covers the color change caused by the reaction of mixtures, was extracted by image processing algorithm including grayscale conversion, thresholding, binarizing, masking, contour detection and noise removal. The extracted ROI area was then masked with original images to calculate average $\mathrm{R}, \mathrm{G}, \mathrm{B}, \mathrm{H}, \mathrm{S}, \mathrm{V}, \mathrm{L}^{*}$, $a^{*}, b^{*}$ values. These values were later used to plot the calibration curve with respect to the concentration values.

\section{Smartphone app: GlucoSense}

In the previous subsection, a calibration curve was extracted by processing the image dataset in a computer. Here, we demonstrate a portable smartphone-based platform for the rapid colorimetric detection of glucose controlled by an application, named as GlucoSense, developed in Android Studio. Contrary to other apps ${ }^{13,15}$ which need an internet connection to transfer the images to process in the workstation, the image-processing algorithm coded in MATLAB was re-coded in Eclipse for the Android platform with the JAVA programming language, resulting in an app capable of image processing without an internet connection (offline). Then, a simple and user-friendly interface was designed to provide rapid on-site quantitative monitoring when a fast analysis is required. Screenshots of the GlucoSense app given in Fig. 2 present the flow of running and quantification procedures. The app acquires images by either accessing the gallery (internal storage) of the smartphone camera, as shown in Fig. 2ii, or using a camera to capture a new 
i.

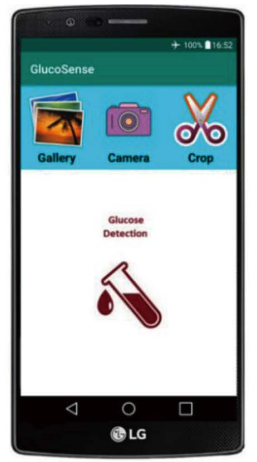

iv.

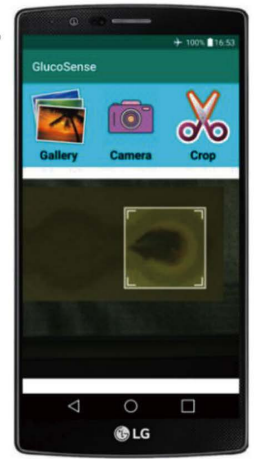

ii.
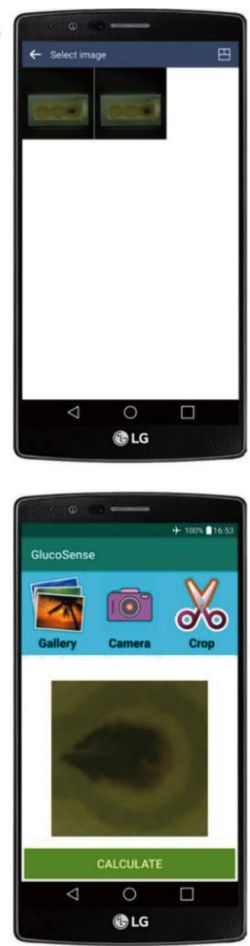

iii.

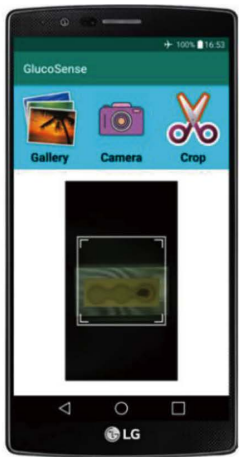

vi.

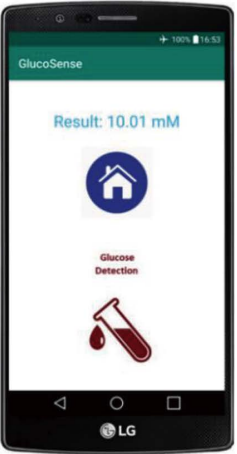

Fig. 2 Steps of colorimetric glucose detection with the GlucoSense app running on an Android smartphone. The homepage of the GlucoSense is given in (i). The user can select an image from the gallery as in (ii) and takes the ROI inside a square to crop a patch as given from (iii) to (v). Once the user taps the "calculate" button in (v), the result is displayed on the screen as shown in (vi).

image. The user takes the ROI inside a square to crop a patch as given from Fig. 2iii to Fig. 2v. Once the user taps the "calculate" button, the image processing algorithm is run on the patch and the ROI is extracted. Average R, V and $\mathrm{L}^{*}$ values in the ROI are used to calculate the glucose concentration of the mixture and the result is displayed on the screen, as shown in Fig. 2vi.

\section{Results and Discussion}

Various 3D-printed stamps were used to cast methacrylic/acrylic resin on both a filter-paper (Fig. $1 \mathrm{C}_{\mathrm{I}}$ ) and a paper-towel (Fig. $1 \mathrm{C}_{\mathrm{II}}$ ) to form hydrophobic channels that control both the direction and the lateral flow of introduced solutions. This approach was facile and cost-effective, and enabled the printing of various shapes quite readily onto a sheet of paper. The resin used for printing was green and hydrophobic in nature. Impression with the self-inking stamp once or twice was enough to form hydrophobic barriers on both types of paper

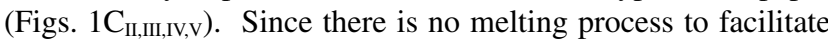
resin penetration into the paper, it is important to see the desired impression on both sides of the sheet prior to light-curing. All processes for a single $\mu$ PAD can be completed in about $30 \mathrm{~s}$, and even less when multiple $\mu$ PADs are made on the same sheet of paper. Next, the lateral flow speed of a colored PBS in $\mu$ PADs made of both types of paper was assessed. Following the injection of $30 \mu \Lambda$ aliquots of colored solution into the insertion region of both devices, the lateral flow was videorecorded with a camera and then analyzed. The observed lateral flow on paper-towel (Fig. 1C $\mathrm{C}_{\text {II }}$ ) was drastically faster than it was on filter paper (Fig. 1 $\mathrm{C}_{\mathrm{I}}$ ). The measured lateral flow speeds on paper-towel and filter paper were 6.3 and $1.2 \mathrm{~mm} / \mathrm{s}$, respectively.
Basically, the paper is made of many small fibers with gaps between them, also referred to as pores. Aqueous solutions are pulled into these pores through capillary action and the motion is further fueled with surface tension. The thickness and average pore size of the filter paper (approx. 180 and $11 \mu \mathrm{m}$, respectively) ${ }^{27}$ are smaller than those of the paper-towel (approx. 240 and $24 \mu \mathrm{m}$, respectively). An increase in the pore size positively influences the fluid flow in a paper. Similarly increasing paper thickness reduces the impact of "edge effect" that hinders the fluid flow. ${ }^{28}$ Due to higher pore size and thickness, the speed of lateral flow on a paper-towel was much higher than that on filter paper, and this was one of the main reasons for choosing paper-towel as a substrate for making $\mu$ PADs. Glucose oxidase/peroxidase method was used for the detection of glucose. The detection mixture comprised GOx, HRP, chitosan and KI. Basically, GOx catalyzes the oxidation of $\beta$-D-glucose to D-glucono-1,5-lactone and also produces $\mathrm{H}_{2} \mathrm{O}_{2}$ as a by-product. ${ }^{29}$ HRP uses the by-product $\mathrm{H}_{2} \mathrm{O}_{2}$ to catalyze the conversion of $\mathrm{KI}$ to iodine and thus produces a visual brown color. Chitosan has been shown to have peroxidase-like activity and to improve the color uniformity and pixel intensity when used with the other components of the detection mixture. ${ }^{10}$ The primary cause of this result may be that the chitosan provides a more suitable micro-environment for direct electron transfer between an enzyme and a reactive surface. The performance of the fabricated $\mu$ PADs in the detection of glucose was determined using a portable smartphone-based platform that included a 3D printed cradle to remove the influence of the ambient light on the analysis. Poor color uniformity especially in lateral flow devices is one of the major problems of this technology. To address this problem, a custom-designed GlucoSense app capable of offline image processing was used to quantify the glucose concentration using a calibration curve extracted by 

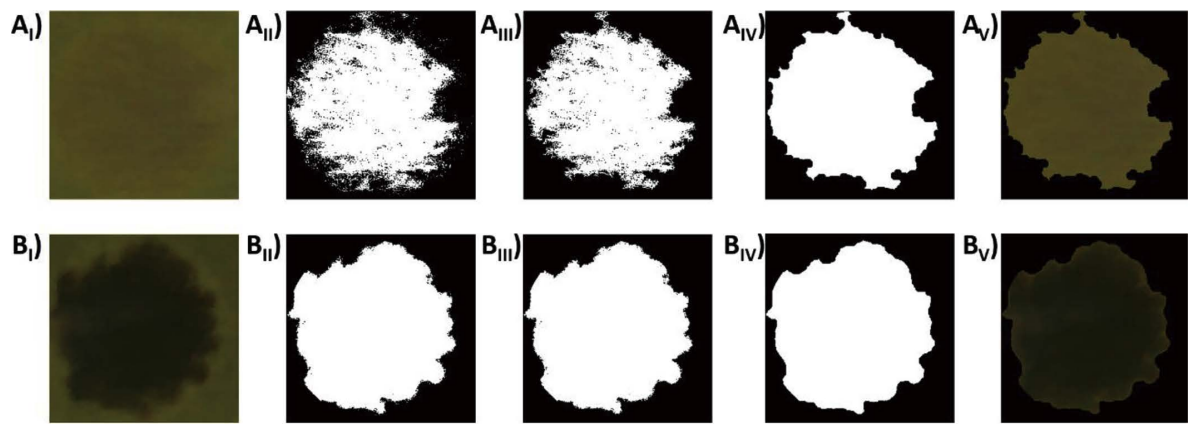

Fig. 3 Image-processing steps for $0.1 \mathrm{mM}$ concentration are given from (a) to (e) while these steps are shown from (f) to (j) for $15 \mathrm{mM}$.

processing the image dataset in MATLAB. Since the app doesn't need internet connection for image processing, the app can be used safely in remote locations. Here, the design given in Fig. $1 \mathrm{C}_{\mathrm{II}}$ was used. Firstly, an image was cropped to decrease the image size, as shown in Figs. $2 \mathrm{iv}$ and $2 \mathrm{v}$. The image was also converted to HSV and $\mathrm{L}^{*} \mathrm{a} * \mathrm{~b} *$ in order to analyze the impact of color spaces on the concentration-depended color change. In the GlucoSense app, the user needs to choose a square patch to maintain consistency in terms of coding (Figs. $2 \mathrm{v}$ and $3 \mathrm{~A}$ ), which needs to be processed with image processing algorithms to find the ROI. First, the square patch is converted to a grayscale image using the green channel of the image. It is then binarized with a threshold value calculated by the Otsu method. ${ }^{30}$ The binarized image was given in Fig. $3 \mathrm{~A}_{\mathrm{II}}$ contained noises (white dots in the black area and black dots in the white area). These noises were removed in two steps by morphological

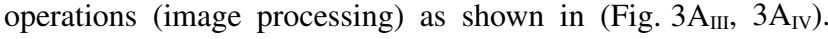
The noise-free image in Fig. $3 \mathrm{~A}_{\mathrm{IV}}$ is then masked with Fig. $3 \mathrm{~A}_{\mathrm{I}}$ to extract the ROI as given in Fig. $3 A_{v}$. These steps are also illustrated in Figs. $3 \mathrm{~B}_{\mathrm{I}}-3 \mathrm{~B}_{\mathrm{V}}$ for $15 \mathrm{mM}$ concentration which has more visible ROI. If the color change in the ROI is sharper, binarized image is less noisy as shown in Fig. $3 \mathrm{~B}_{\mathrm{II}}$. After the ROI is extracted, average R, G, B values are calculated. The same ROI is applied to HSV and L*a*b* images resulting in nine color variables to analyze color change depending on the concentration variation.

The overall process given above was also employed in the RAW images. The calibration curves were obtained based on average $\mathrm{R}, \mathrm{G}, \mathrm{B}, \mathrm{H}, \mathrm{S}, \mathrm{V}, \mathrm{L}^{*}, \mathrm{a}^{*}, \mathrm{~b}^{*}$ values in the ROI as given in Fig. 4. Based on experimental investigations, the average of $\mathrm{R}, \mathrm{V}$ and $\mathrm{L}^{*}$ gives more reliable characteristics as $\mathrm{V}$ and $\mathrm{L}^{*}$ are robust to illumination change. Calibration curves were obtained by processing images both in RAW and JPEG formats to analyze the impact of formats on quantitative evaluation. The relationship between the normalized intensity and the glucose concentration was linear in the range of 0 to $1 \mathrm{mM}$ of glucose in both image formats. $R^{2}$ of the calibration curve obtained using JPEG was 0.9949 (Fig. 4A $\mathrm{A}_{\mathrm{I}}$ ) was slightly better than that obtained using RAW images $\left(R^{2}: 0.9767\right)$. Therefore, the JPEG image format can be used to adequately quantify the glucose concentration by the user as it is the most supported image format in smartphones and easy to process. It might be difficult for end users to choose the right region for more accurate analysis. To demonstrate the utility of the image processing tool, calibration curves of the same dataset were obtained by manual selection of the ROI. All circle in detection zone was considered as the ROI since manual detection of the color change area by the user will be time-consuming which leads the user to choose all circle instead of pointing the colored area in detail. As can be seen in Fig. $4 \mathrm{~A}_{\mathrm{I}}$, the image processing tool improved the accuracy and thus the sensitivity of the measurement considering the change in the slopes of the calibration curves obtained between 0 and $1 \mathrm{mM}$ of glucose (red circles: with image processing, blue circles: without image processing). Due to the higher water absorption efficiency of paper-towels, the color changing intensity remained almost constant for all test solutions only $30 \mathrm{~s}$ after glucose addition to the sample zone, which significantly shortens the assay time (less than one minute) considering some recently published similar papers ${ }^{31-35}$ (Fig. $4 \mathrm{~A}_{\mathrm{II}}$ ). The analysis with filter-paper based $\mu$ PADs is usually completed within 10 to $20 \mathrm{~min}^{36-38}$ In addition, each $\mu$ PADs can be produced at less than $\$ 0.2$. Various 3D-printed stamps can be used to make different $\mu$ PADs for different applications.

Here, a $\mu$ PAD with smaller size and different design (Fig. $1 \mathrm{C}_{\mathrm{IV}}$ ) was also designed for measuring glucose in a $10 \mu \mathrm{L}$ of an artificial saliva droplet. This design could be more suitable for the detection of glucose in saliva as the sample insertion zone can be placed on the tongue for saliva suction (Fig. $4 \mathrm{~B}_{\text {III }}$ ). Due to its small size, $2 \mu \mathrm{L}$ aliquots of the detection mixture were carefully and slowly injected into the detection zone of $\mu$ PADs and allowed to dry at room temperature $(10 \mathrm{~min})$. The artificial saliva droplets contained glucose at varying concentrations $(0.1$, $0.25,0.5,0.75$, and $1 \mathrm{mM}$ ). Simply, the solution absorption region of the $\mu \mathrm{PAD}$ was touched to the droplet and the solution was sucked up through the channel and delivered to the detection zone through capillary force without the use of external equipment. A visible color was observed as soon as the sample solution reached the detection zone. The sensor gave a linear response for a glucose concentration range of between 0.1 and $1 \mathrm{mM}\left(R^{2}=0.9819\right)$ in artificial saliva with a calculated LOD of $29.65 \mu \mathrm{M}$. The LOD of the device appears to be lower than that of $\mu \mathrm{PADs}{ }^{11,31,38-42}$ reported in the literature, especially those using $\mathrm{KI}$ as an indicator (Fig. $4 \mathrm{~B}_{\mathrm{I}}$ ). As mentioned above, the image processing tool of the smartphone positively influenced the sensitivity of the measurement, thus leading to a lower LOD. Although the linear response of the device seems to be narrower than these reported studies, it is linear in a lower glucose concentration range. This makes it more suitable for the detection of glucose in other bodily fluids, such as saliva, where the glucose concentration is lower than that of blood. ${ }^{43,44}$ The selectivity of the sensor was also tested against some interfering species and the results showed that the color intensity in the presence of these species was comparable to that of saliva with no glucose (Fig. 4B $\mathrm{B}_{\mathrm{II}}$ ). 

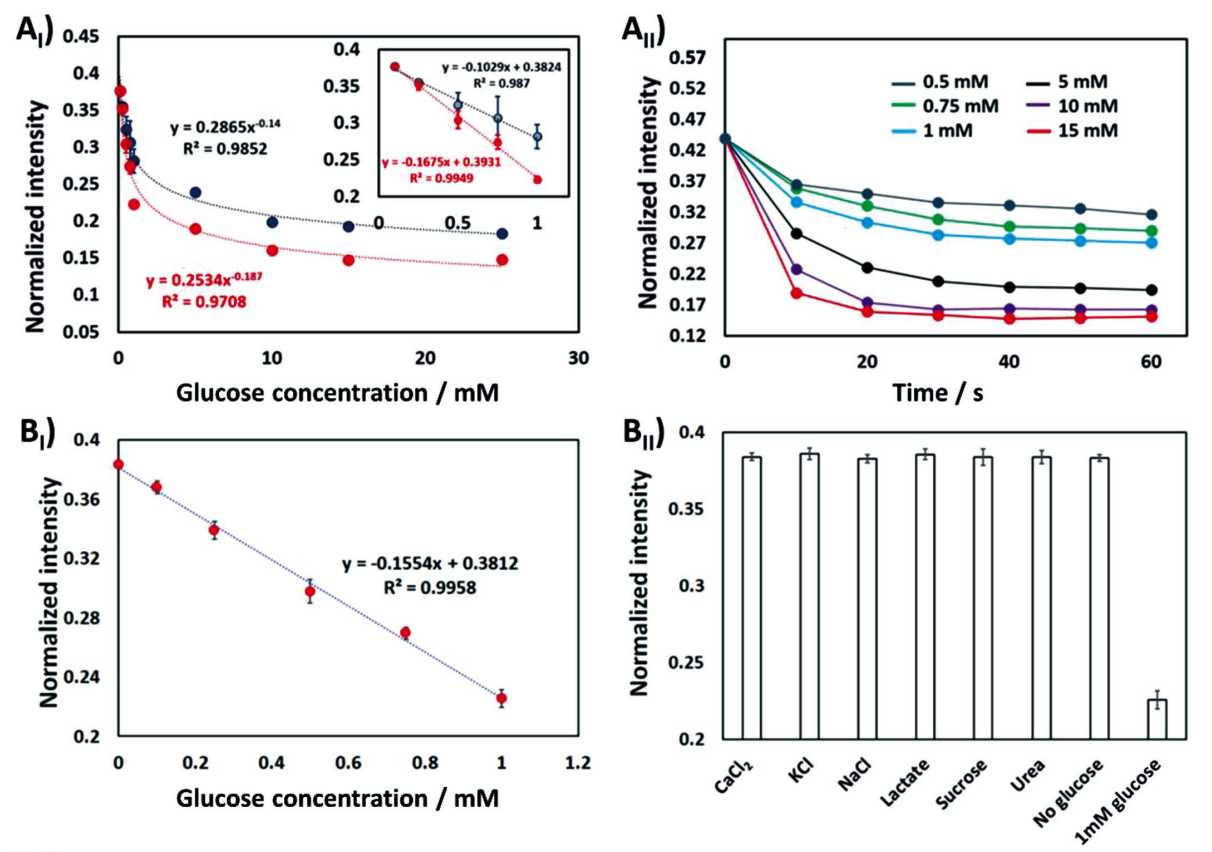

$\left.B_{\text {IIII }}\right)$
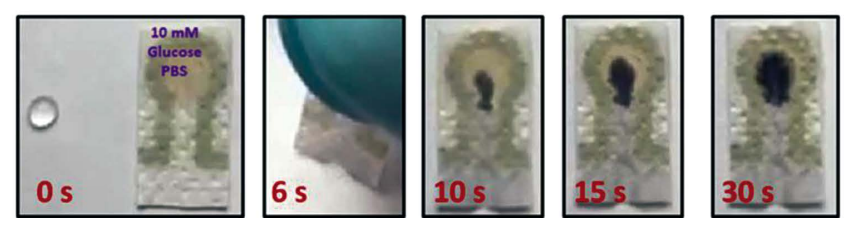

Fig. 4 Glucose concentration-dependent intensity (normalized) calibration curves obtained with (red circles) and without (blue circles) image processing tool using JPEG $\left(\mathrm{A}_{\mathrm{I}}\right)$ images $(n=3)$. Timedependent color intensity (normalized) change of different glucose concentration values $\left(\mathrm{A}_{\mathrm{II}}\right)$. The sensitivity $\left(\mathrm{B}_{\mathrm{I}}\right)$ and selectivity $\left(\mathrm{B}_{\mathrm{II}}\right)$ of the sensor were assessed in artificial saliva containing glucose from 0 to 1 and $20 \mathrm{mM}$ interferents, respectively. A $\mu$ PAD designed to measure glucose concentration in a saliva droplet $\left(\mathrm{B}_{\mathrm{III}}\right)$.

\section{Conclusions}

Inthe present work, a $\mu$ PAD was integrated with a portable smartphone-based platform for rapid, sensitive, selective and quantitative detection of glucose in PBS first and then artificial saliva. The platform is user-friendly, ultra-low cost and fielddeployable. A light curable polymer was successfully used to form hydrophobic channels on paper. A custom-designed 3D printed case with a smartphone was used for imaging to eliminate ambient light interference. Images were saved both in JPEG and RAW formats. All captured images were processed in MATLAB for precise extraction of the ROI to address one of the major limitations of this technology which is poor color uniformity and thus to improve the accuracy of the analysis. The obtained data were used to obtain calibration curves. According to the results, both image formats can be used successfully for quantitative analysis due to the negligible differences. Due to the higher water absorption efficiency of paper-towels, the color changing intensity remained almost constant for all test solutions only $30 \mathrm{~s}$ after glucose addition to the sample insertion zone, which enabled the completion of each test in less than $1 \mathrm{~min}$. An Android app, named GlucoSense, was developed by re-coding all image processing algorithms in JAVA to make the platform more user-friendly and allow the users to make offline quantitative glucose analysis. The app will be further improved to include various capabilities such as user-oriented self-calibration and running on ios platform.
A different $\mu$ PAD design was also used for glucose detection in artificial saliva to demonstrate that the proposed strategy can be adapted for various applications; the sensor demonstrated a linear response for glucose ranging between 0 and $1 \mathrm{mM}$ with a calculated detection limit of $29.65 \mu \mathrm{M}$. The integrated platform has great potential for non-invasive measurement of glucose in body fluids like tear, sweat and saliva, where the glucose concentration is much lower.

\section{Acknowledgements}

This research was supported by the scientific research projects coordination unit of Izmir Katip Celebi University (project no. 2019-ÖNAP-MÜMF-0004 and 2018-ÖDL-MÜMF-0021) and partly supported by the Scientific and Technical Research Council of Turkey (project Nos. 116E934 and 215E003).

\section{References}

1. T. Akyazi, L. Basabe-Desmonts, and F. Benito-Lopez, Anal. Chim. Acta, 2018, 1001, 1.

2. H. Kettler, K. White, and S. J. Hawkes, "Mapping the landscape of diagnostics for sexually transmitted infections: key findings and recommendations", 2004, World Health Organization.

3. D. M. Cate, J. A. Adkins, J. Mettakoonpitak, and C. S. 
Henry, Anal. Chem., 2015, 87, 19.

4. A. W. Martinez, S. T. Phillips, M. J. Butte, and G. M. Whitesides, Angew. Chem. Int. Ed., 2007, 119, 1340.

5. H. Asano and Y. Shiraishi, Anal. Sci., 2018, 34, 71.

6. L. S. A. Busa, M. Maeki, A. Ishida, H. Tani, and M. Tokeshi, Sens. Actuators, B, 2016, 236, 433.

7. Y. Lin, D. Gritsenko, S. Feng, Y. C. Teh, X. Lu, and J. Xu, Biosens. Bioelectron., 2016, 83, 256.

8. T. Komatsu, M. Maeki, A. Ishida, H. Tani, and M. Tokeshi, Anal. Sci., 2018, 34, 39.

9. M. Salles, G. Meloni, W. De Araujo, and T. Paixão, Anal. Methods, 2014, 6, 2047.

10. E. F. M. Gabriel, P. T. Garcia, T. M. G. Cardoso, F. M. Lopes, F. T. Martins, and W. K. T. Coltro, Analyst, 2016, $141,4749$.

11. P. T. Garcia, T. M. G. Cardoso, C. D. Garcia, E. Carrilho, and W. K. T. Coltro, RSC Adv., 2014, 4, 37637.

12. E. Evans, E. F. M. Gabriel, W. K. T. Coltro, and C. D. Garcia, Analyst, 2014, 139, 2127.

13. H. Kim, O. Awofeso, S. Choi, Y. Jung, and E. Bae, Appl. Opt., 2017, 56, 84.

14. V. Kılıç, N. Horzum, and M. E. Solmaz, "Color Detection", 2018, IntechOpen.

15. M. E. Solmaz, A. Y. Mutlu, G. Alankus, V. K1lıç, A. Bayram, and N. Horzum, Sens. Actuators, B, 2018, 255, 1967.

16. G. K. Özdemir, A. Bayram, V. K1lıç, N. Horzum, and M. E. Solmaz, Anal. Methods, 2017, 9, 579.

17. S. Schaefer, "Colorimetric water quality sensing with mobile smartphones", 2014, University of British Columbia.

18. S. Dutta, D. Sarma, A. Patel, and P. Nath, IEEE Photon. Technol. Lett., 2015, 27, 2363.

19. Y. Wang, X. Liu, P. Chen, N. T. Tran, J. Zhang, W. S. Chia, S. Boujday, and B. Liedberg, Analyst, 2016, 141, 3233.

20. A. Bayram, N. Horzum, A. U. Metin, V. Kılıç, and M. E. Solmaz, IEEE Sens. J., 2018, 18, 5948.

21. A. F. Coskun, J. Wong, D. Khodadadi, R. Nagi, A. Tey, and A. Ozcan, Lab Chip, 2013, 13, 636.

22. Y. Jung, J. Kim, O. Awofeso, H. Kim, F. Regnier, and E. Bae, Appl. Opt., 2015, 54, 9183.

23. C. K. Kuşçuoğlu, H. Güner, M. A. Söylemez, O. Güven, and M. Barsbay, Sens. Actuators, B, 2019, 296, 126653.

24. L. Shen, J. A. Hagen, and I. Papautsky, Lab Chip, 2012, 12,
4240.

25. A. K. Yetisen, J. Martinez-Hurtado, A. Garcia-Melendrez, F. da Cruz Vasconcellos, and C. R. Lowe, Sens. Actuators, $B, \mathbf{2 0 1 4}, 196,156$.

26. D. Coffin, Decoding raw digital photos in Linux, 2016.

27. Whatman qualitative filter paper, Grade 1, https://www. sigmaaldrich.com/catalog/product/aldrich/wha1001125?lang= en\&region=TR.

28. V. Soum, S. Park, A.I. Brilian, O.S. Kwon, and K. Shin, Micromachines, 2019, 10, 516.

29. V. K. Aydin and M. Şen, J. Electroanal. Chem., 2017, 799, 525.

30. H. J. Vala and A. Baxi, Int. J. Adv. Res. Comput. Eng. Technol., 2013, 2, 387.

31. P. Martinkova, M. Brtnicky, J. Kynicky, and M. Pohanka, Chem. Zvesti, 2018, 72, 2719.

32. Y. Li, J. Sun, W. Mao, S. Tang, K. Liu, T. Qi, H. Deng, W. Shen, L. Chen, and L. Peng, Microchim. Acta, 2019, 186, 403.

33. H. Zhang, E. Smith, W. Zhang, and A. Zhou, Biomed. Microdevices, 2019, 21, 48.

34. F. Li, X. Wang, J. Liu, Y. Hu, and J. He, Sens. Actuators, B, 2019, 288, 266.

35. N. Alizadeh, A. Salimi, and R. Hallaj, Sens. Actuators, B, 2019, 288, 44.

36. M.-Y. Jia, Q.-S. Wu, H. Li, Y. Zhang, Y.-F. Guan, and L. J. B. Feng, Biosens. Bioelectron., 2015, 74, 1029.

37. X. Wang, F. Li, Z. Cai, K. Liu, J. Li, B. Zhang, and J. He, Anal. Bioanal. Chem., 2018, 410, 2647.

38. M.-M. Liu, X. Lian, H. Liu, Z.-Z. Guo, H.-H. Huang, Y. Lei, H.-P. Peng, W. Chen, X.-H. Lin, and A.-L. Liu, Talanta, 2019, 200, 511.

39. D. Basş, Anal. Methods, 2017, 9, 6698.

40. E. Evans, E. F. M. Gabriel, T. E. Benavidez, W. K. T. Coltro, and C. D. Garcia, Analyst, 2014, 139, 5560.

41. G. Demirel and E. Babur, Analyst, 2014, 139, 2326.

42. S. Oyola-Reynoso, A. P. Heim, J. Halbertsma-Black, C. Zhao, I. D. Tevis, S. Çınar, R. Cademartiri, X. Liu, J. F. Bloch, and M. M. Thuo, Talanta, 2015, 144, 289.

43. A. Takahiro, Y. Kuroki, H. Nitta, P. Chouhan, K. Toma, S. Sawada, S. Takeuchi, T. Sekita, K. Akiyoshi, S. Minakuchi, and K. Mitsubayashi, Biosens. Bioelectron., 2016, 84, 106. 44. S. Jang and C. Xu, Int. J. Biosens. Bioelectron., 2018, 4, 5. 